\title{
Zone II Flexor Tendon Repair in a 13-Month-Old: Report of a Complication
}

\author{
Joao B. Panattoni, Mohammed M. Ahmed* \\ Department of Orthopaedic Surgery, School of Medicine, Saint Louis University, Saint Louis, USA. \\ Email: mahmed9@slu.edu
}

Received December $1^{\text {st }}, 2013$; revised December $29^{\text {th }}, 2013$; accepted January $9^{\text {th }}, 2014$

Copyright (C) 2014 Joao B. Panattoni, Mohammed M. Ahmed. This is an open access article distributed under the Creative Commons Attribution License, which permits unrestricted use, distribution, and reproduction in any medium, provided the original work is properly cited. In accordance of the Creative Commons Attribution License all Copyrights (C) 2014 are reserved for SCIRP and the owner of the intellectual property Mohammed M. Ahmed Joao B. Panattoni. All Copyright (C) 2014 are guarded by law and by SCIRP as a guardian.

\section{ABSTRACT}

Despite early cautions against the primary repair of zone II flexor tendon injuries, recent advances in surgical technique and suture materials have allowed such repairs to become commonplace. The 6-strand repair technique is rarely applied to the young pediatric population, however, to our knowledge, no English-language articles have described this method of primary repair in zone II of children less than 2 years old. A 13-month-old male presented flexor digitorum profundus repair after lacerating it in zone II on a sharp aluminum can. The tendon was repaired with a 6-strand technique, using a 4.0 Fiberloop for the core suture and 6.0 Prolene for the epitendinous suture. Approximately four months after surgery, the patient developed a palmar collection at the level of his middle phalanx and a serosanguinous sinus tract at the distal interphalangeal crease. During the revision surgery, the inspection of the repaired tendon revealed a small gap filled with scar tissue. There was no evidence of new fistula formation at his final visit one month after the second procedure. After the revision, the patient could move his digit with minimal loss of range of motion at the distal interphalangeal joints. Unfortunately, he was subsequently lost to follow up. This surgical technique was selected to provide a strong repair that would allow the early postoperative movement. In retrospect, a 6-strand repair with braided suture is not ideal in young children as the bulky suture can cause a foreign-body reaction and possibly extrude through the skin. Additionally, the immobilization with a long-arm cast remains a valuable tool after tendon repair in infants who cannot voluntarily restrict their movements.

\section{KEYWORDS}

Flexor Tendon Repair; Pediatrics; Complications

\section{Introduction}

Flexor tendon injuries of the hand are common among adults, with an incidence of about 15,000 cases per year in the United States [1]. Not surprisingly, the literature is rich with information describing surgical techniques and outcomes, and several additional studies investigating novel techniques and materials are currently underway [24]. However, many common techniques used to enhance postoperative tendon strength in the adult population have not yet been applied to young pediatric patients. Specifically, to our knowledge, this is the first Englishlanguage report of a 6-strand zone II repair with locking

\footnotetext{
"Corresponding author.
}

techniques in a patient younger than 2 years old.

Modern surgical techniques used in flexor tendon repairs have evolved from procedures originally developed over sixty-five years ago. In his pioneering publication "Surgery of the Hand," Sterling Bunnell advocated the use of the delicate instrumentation with the atraumatic technique [5]. He expounded on the importance of avoiding longitudinal incisions, preserving pulleys, maintaining tension on the repair, and allowing early guarded motion. These recommendations served as guidelines and were adopted internationally for many years. Additionally, Bunnell was known for cautioning against the primary repair of tendons in zone II, which he described as "no-man’s land" [5]. Instead, he recommended a 2-stage 
repair by silicone rod placement in all zone II flexor tendon injuries.

Concerned by the sub-optimal results with his zone II repairs, Claude Verdan, in 1960, questioned Bunnell's recommendation and began attempting primary repair on zone II injuries [6]. His method called for resection of the flexor tendon sheath and superficialis tendon, with the use of transfixion pins. Seven years later, Harold Kleinert revolutionized the indications for zone II flexor tendon repair, and his meticulous technique helped him achieve $74 \%$ good-to-excellent results, compared to poor outcomes in $76 \%$ of cases on most teaching services throughout the US at that time $[7,8]$.

Primary repair in zone II followed by early range of motion therapy was supported by a series of laboratory experiments performed by Gelberman and colleagues. Using canine models, he demonstrated that passive mobilization enhances healing by stimulating maturation of the tendon wound simultaneously with remodeling of the tendon scar [9]. It was also demonstrated that the strength of the repair increases proportionally to the number of strands used in the surgical reconstruction, a crucial finding before stronger suture materials became available $[10,11]$. With stronger materials, the predominant mode of failure shifted from suture rupture to pullout from the tendon [12]. Locking loop suture configurations were developed to combat suture pullout by increasing grip on the tendon [13].

Despite these substantial advances, problems persisting as zone II repairs continue to present with the potential for adhesions, rupture, and poor functional outcome. Additionally, the pediatric population is impacted by two unique challenges. Firstly, the pediatric hand structures are very small relative to the repair materials, such that it is difficult for the flexor tendon to accommodate the necessary number of strands with locking. This can lead to tightening of the fibro-osseous sheath at zone II [14]. Secondly, young pediatric patients are frequently uncooperative and may not be able to maintain postoperative immobilization [15].

We attempted to overcome the challenges of pediatric flexor tendon repair with loop sutures and locking techniques in order to optimize a rapid return to active mobility. The purpose of this report is to describe the outcome of a multi-strand flexor tendon repair in zone 2 of a 13 month-old patient designed to maximize strength for early, active mobility. Special attention will be given to the surgical technique and complications encountered.

\section{Case Report}

A 13-month-old male accidentally lacerated his left index finger (Figure 1) when he fell while holding a sharp aluminum can. Delayed primary repair was performed 11

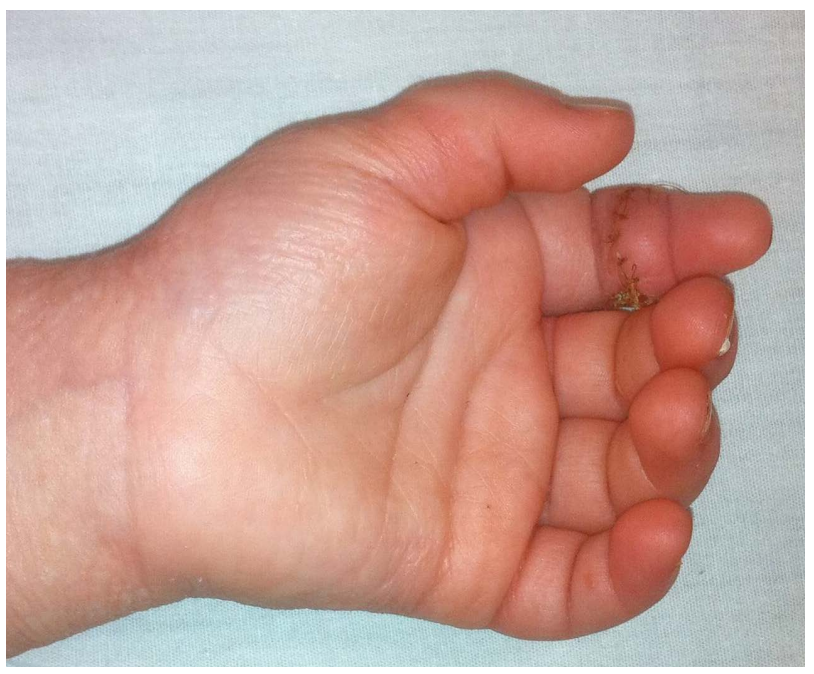

Figure 1. The patient's injury at initial presentation.

days after injury at the Cardinal Glennon Children's Medical Center in Saint Louis, MO, and a second procedure was performed five months after the first operation due to the extrusion of suture material. Postoperative follow-up continued for one month after the second procedure, at which time the patient relocated out of state.

\section{Treatment}

Surgical incision followed Brunner's zigzag approach [16]. An atraumatic technique was used with careful respect to the anatomy and gentle manipulation of the tissues. The flexor digitorum profundus and ulnar digital nerve were both $100 \%$ lacerated, but the flexor digitorum superficialis remained intact (Figure 2). One centimeter of the tendon's distal stump was available for suture at the transition between zones I and II. The tendon was repaired under loupe magnification (3.5×) with a 6-strand technique, using a 4.0 Fiberloop (Arthrex, Inc. Naples, FL) for the core suture and 6.0 running Prolene (Ethicon, Inc. Somerville, NJ) for the epitendinous suture [17]. The nerve was repaired with a 10.0 nylon suture under microscope. After careful hemostasis was achieved, the wound was closed with 5 - 0 chromic. A dorsal blocking long-arm splint extending to the fingertips was applied with the wrist in 20 degrees of extension and the metacarpophalangeal joints in 60 degrees of flexion.

Per the patient's father, the patient removed his splint on the 14th postoperative day shortly before his first follow-up appointment. At that time, the patient presented with mild erythema in his index finger and two small blisters volarly on the middle phalanx and distal interphalangeal crease (Figure 3). Daily soapy soaks were initiated and prophylactic oral antibiotics were prescribed for one week. No further immobilization was applied, and the patient began early rehabilitation therapy with 


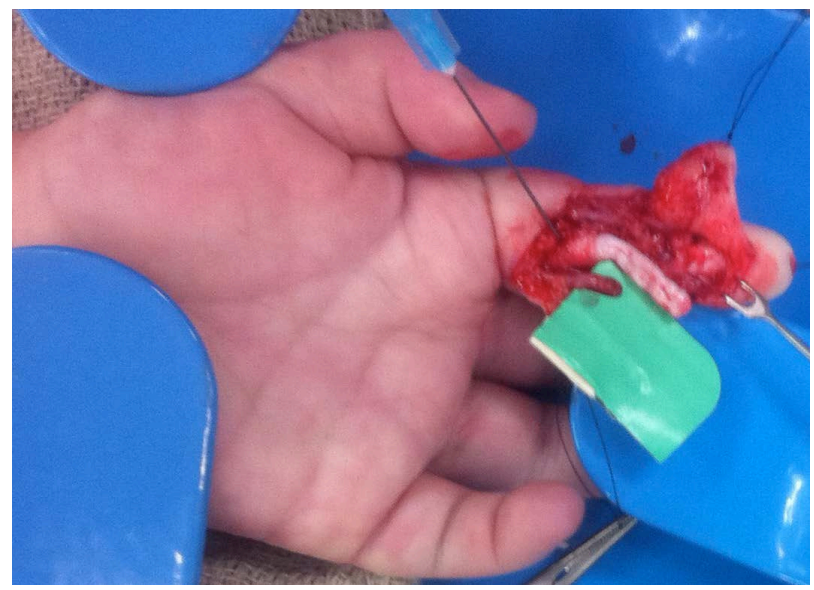

Figure 2. Intraoperative examination revealed complete laceration of the patient's flexor digitorum profundus and ulnar digital nerve.

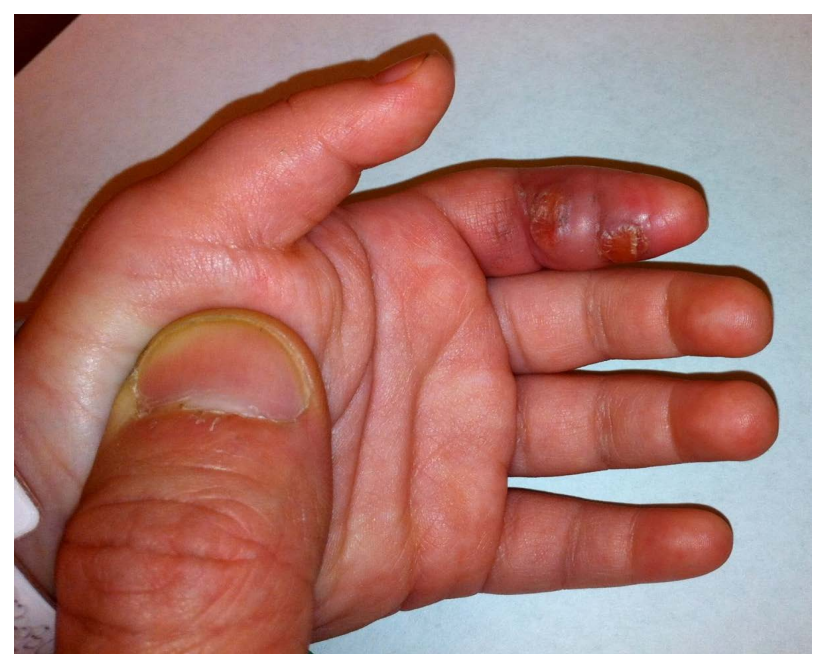

Figure 3. The wound appears to be healing well 2 weeks postoperatively, with mild swelling and erythema.

active and passive range of motion exercises, edema control, and scar massage. The wound healed with a minimal amount of residual swelling and erythema.

\section{Complication}

Approximately four months after surgery, the Occupational Therapist noted a palmar collection at the level of the middle phalanx and an oozing, scabbed wound with sanguineous secretions at the distal interphalangeal crease (Figure 4). The patient, however, was actively using his finger and his father noticed that he had improved sensation. The patient was closely followed and the wounds developed into a draining sinus with serous secretions. According to his parents, the sinus tract occasionally closed followed by increased swelling in the finger. Our initial impression of a chronic wound due to an insensate finger was no longer valid as it was nearly

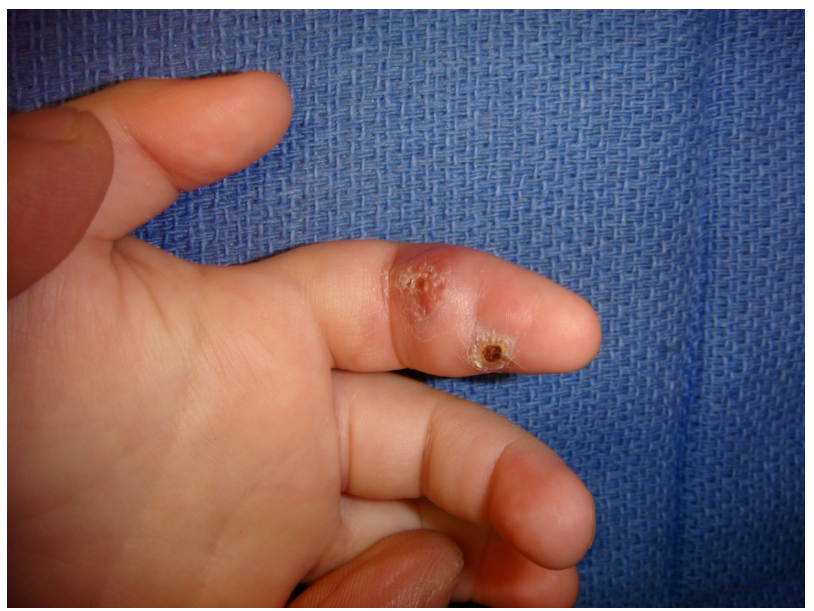

Figure 4. Four months postoperatively the patient developed a palmar collection and serosanguinous drainage near his middle phalanx.

five months after surgery and the patient was showing some sensation to touch on his finger. With the diagnosis of a foreign body reaction, surgery was scheduled for tendon exploration and removal of suture material.

On the second surgery, we observed that the distal anchoring knot's suture material (Fiberloop) was extruding into the skin (Figures 5 and 6). All suture materials were removed without complications. We also observed some gapping of the tendon filled with scar tissue. Some tendon adhesion was observed distally and tenolysis was performed (Figure 7). Subsequently the A4 pulley appeared to be insufficient with bowstringing of the tendon, so the pulley was reconstructed using the ulnar band of the flexor digitorum superficialis [18,19] (Figure 8). Irrigation and hemostasis were performed and the skin was closed with absorbable sutures (5 - 0 chromic). The patient was placed into a long arm splint.

\section{Postoperative Course}

On his first postoperative visit at two weeks, the surgical wound appeared to be healing well (Figure 9). The patient's father reported that the patient had removed his splint two days after surgery. On examination, the patient was actively using his fingers without appreciable pain. The patient was prescribed occupational therapy. On his last follow up visit approximately one month after his second surgery, no new fistula formation was observed. The patient was actively moving his finger with minimally decreased range of motion at the distal interphalangeal joint and no pain on passive range of motion.

Unfortunately, the patient was lost to follow up after moving to another state. Before leaving, the family was instructed to continue their home exercises and look for specialized follow up care in their new location. Instructions about prognosis and possible future complications, 


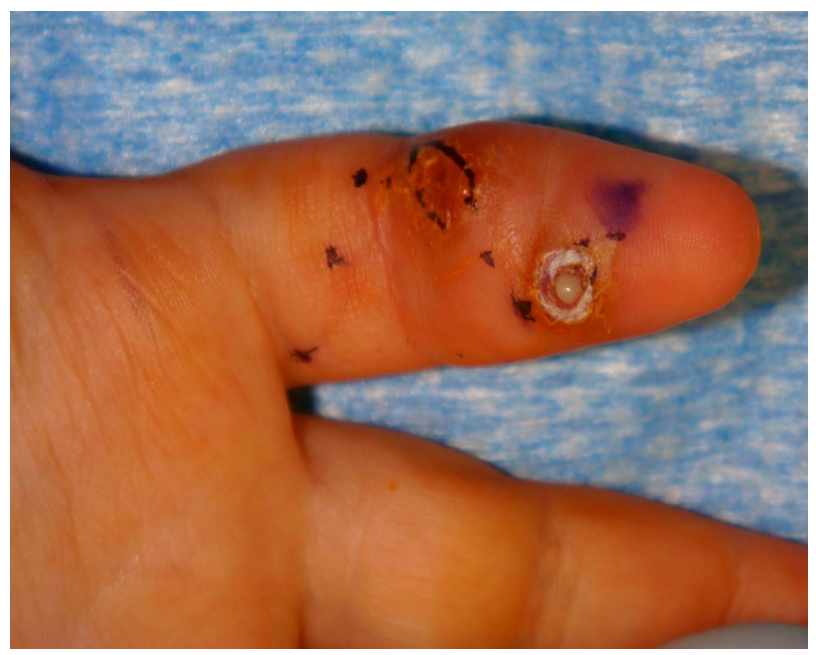

Figure 5. A closer view of the patient's draining sinus.

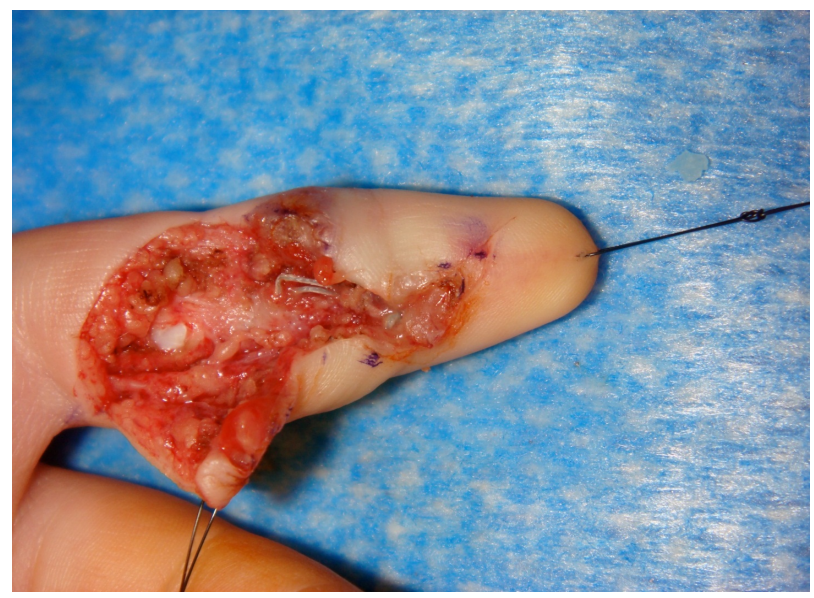

Figure 6. Intraoperative examination revealed suture material protruding into the sinus.

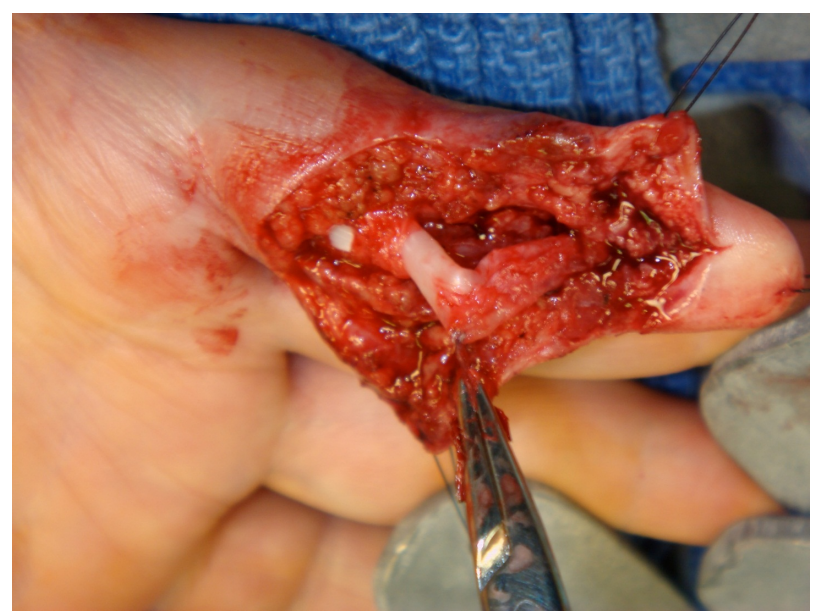

Figure 7. Tenolysis.

such as scar retraction and delayed growth, were given. Attempts to contact the family by email were unsuccessful.

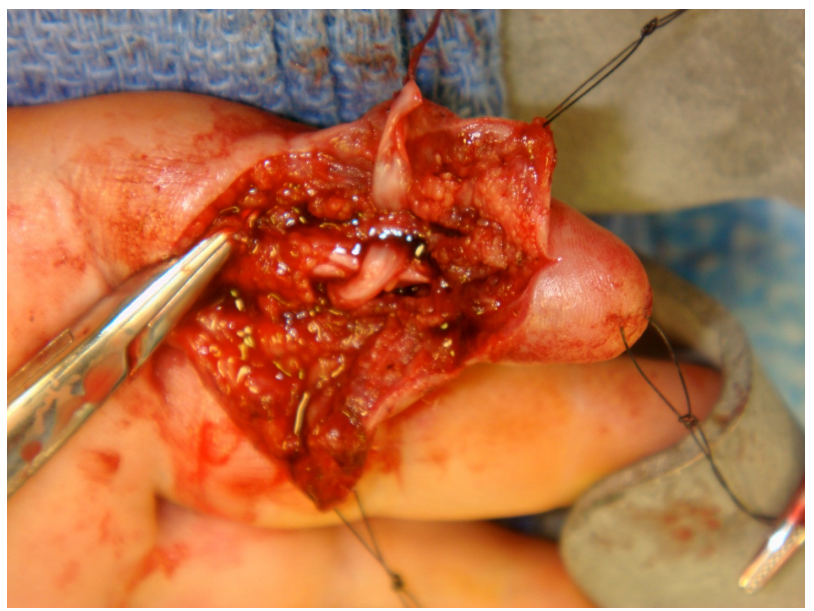

Figure 8. Pulley reconstruction.

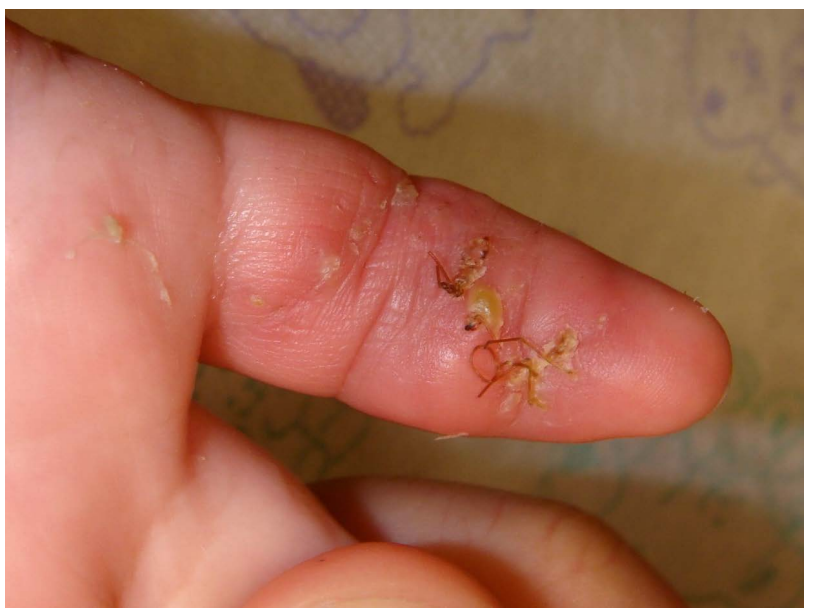

Figure 9. The wound appears to be healing well one month after the second operation.

\section{Discussion}

Pediatric flexor tendon injury repair continues to pose a substantial challenge to hand surgeons, primarily due to the small anatomic structures and the fact that young patients may not be able to follow recommendations to avoid early forceful gripping.

As the patient's flexor digitorum profundus was injured just one centimeter from its insertion, standard procedures call for anchoring with a pullout suture [19]. We decided to perform a 6-strand tendon repair instead in order to avoid potential nail deformities and discomfort upon the suture's removal. Additionally, we anticipated that the child would actively move his hand early in the postoperative period and we assumed that a locking technique would prevent gapping.

However, the second surgery revealed some gapping filled with scar tissue, proving that the suture was insufficient to resist a pull out at the distal part of the tendon. Given the fact that gapping developed with a 6-strand 
technique, it is important to prioritize the use of the pullout suture when the distal stump of the tendon is short. As an additional benefit in this case, the pullout suture would have allowed us to avoid placing a knot near the fingertip where the soft tissue envelope is more constricting.

In our opinion, a strong suture material and 6-strand repair may not be ideal for very young patients. The bulkier braided suture can extrude through the skin of a small finger, causing a local foreign body reaction and a non-healing ulcer. Instead, a monofilament 2-strand technique might be preferable in infants [20]. Previous reports showed that the two-strand core suture with 4 - 0 nylon using the modified Kessler technique is strong enough to avoid tendon rupture when the finger is immobilized for 3 to 4 weeks after surgery $[15,21]$.

Previous reports claim no benefits of early mobilization protocols in children, with no influence on ultimate digital performance when immobilization is maintained up to four weeks after the operation [22]. However, for the present case, an early mobilization protocol was chosen for the following reasons: 1) anticipation of patient low compliance (as evidenced by the patient's removal of the immobilization in the early postoperative period after both surgical procedures), 2) comfort of the patient (avoidance of a long arm cast), and 3) the assumption that a 6-strand locking repair with strong suture would be reliable enough to permit early mobilization. This case demonstrates that despite the low compliance that is expected among young patients, a longer immobilization with long-arm cast is still a valuable tool after repair of tendon injuries in infants. It is possible that a very close (weekly) follow up would help to prevent precocious removal of the immobilization.

\section{Conclusion}

Patient selection remains important despite advances in the suture materials and techniques used for flexor tendon repair. Specifically in the pediatric population, a bulkier, braided suture with 6-strands seems suboptimal in patients younger than two years old due to the risk of suture material extrusion. The classic 2-strand monofilament suture technique with up to four weeks of postoperative immobilization has stood over time and should be considered in the repair of zone II flexor tendon injuries in young children. The pullout suture is also a valuable tool that should be considered at the more distal zone II injuries. Selection of proper suture material and surgical technique is critical in achieving a strong and uncomplicated repair.

\section{REFERENCES}

[1] R. A. E. Clayton and C. M. Court-Brown, "The Epidemi- ology of Musculoskeletal Tendinous and Ligamentous Injuries,” Injury, Vol. 39, No. 12, 2008, pp. 1338-344. http://dx.doi.org/10.1016/j.injury.2008.06.021

[2] M. A. Badalamente and E. Wang, "Collagenase in the Treatment of Zone II Flexor Tendon Adhesions in the Hand,” In: Clinical Trials.gov [Internet], National Library of Medicine (US), Bethesda.

http://www.clinicaltrials.gov/ct2/show/NCT00261209?ter $\mathrm{m}=$ NCT00261209\&rank=1

[3] I. R. MacArthur, S. Logsetty and L. J. Sigurdson, “The Effects of a Stainless Steel Suture MGH Flexor Tendon Repair Coupled with Early Aggressive Range of Motion Rehabilitation: A Randomized Controlled Trial,” In: ClinicalTrials.gov [Internet]. National Library of Medicine (US), Bethesda.

http://www.clinicaltrials.gov/ct2/show/NCT01344980?ter $\underline{\mathrm{m}=\text { NCT01344980\&rank=1 }}$

[4] G. Andreisek, "FLEX-Trial: Prospective Sonographic Assessment of Healing Process Following Suture of Profound Flexor Tendon Due to Traumatic Rupture of FDPTendon in Zone II,” In: ClinicalTrials.gov [Internet]. National Library of Medicine (US), Bethesda (MD).

http://www.clinicaltrials.gov/ct2/show/NCT01013428?ter $\underline{\mathrm{m}=\text { NCT01013428\&rank=1 }}$

[5] S. Bunnell, "Surgery of the Hand," 3rd Edition, J. B. Lippincott Company, Philadelphia, 1948.

[6] C. E. Verdan, "Primary Repair of Flexor Tendons," The Journal of Bone \& Joint Surgery, Vol. 42A, 1960, pp. 123-127.

[7] H. E. Kleinert, J. E. Kutz, T. S. Ashbell and E. Martinez, "Primary Repair of Lacerated Flexor Tendons in 'No Man's Land'," The Journal of Bone \& Joint Surgery, Vol. 49A, 1967, p. 577.

[8] G. D. Lister, H. E. Kleinert, J. E. Kutz and E. Atasoy, "Primary Flexor Tendon Repair Followed by Immediate Controlled Mobilization,” The Journal of Hand Surgery, Vol. 2, No. 6, 1977, pp. 441-451.

[9] J. W. Strickland, "Development of Flexor Tendon Surgery: Twenty-Five Years of Progress," The Journal of Hand Surgery, Vol. 25, No. 2, 2000, pp. 214-235. http://dx.doi.org/10.1053/jhsu.2000.0214

[10] R. T. Thurman, T. E. Trumble, D. P. Hanel, A. F. Tencer and P. K. Kiser, "Two-, Four- and Six-Strand Zone II Flexor Tendon Repairs: An in Situ Biomechanical Comparison Using a Cadaver Model," The Journal of Hand Surgery, Vol. 23, No. 2, 1998, pp. 261-265. http://dx.doi.org/10.1016/S0363-5023(98)80124-X

[11] B. H. Lim and T. M. Tsai, "The Six-Strand Technique for Flexor Tendon Repair,” Atlas of The Hand Clinics, Vol. 1, 1996, pp. 65-76.

[12] B. Miller, S. D. Dodds, A. deMars, N. Zagoreas, T. Waitayawinyu and T. E. Trumble, "Flexor Tendon Repairs: The Impact of Fiberwire on Grasping and Locking Core Sutures,” The Journal of Hand Surgery, Vol. 32, No. 5, 2007, pp. 591-596. http://dx.doi.org/10.1016/j.jhsa.2007.03.003

[13] T. Karjalainen, M. He, A. K. Chong, A. T. Lim and J. Ryhanen, "An Analysis of the Pull out Strength of 6 Suture Loop Configurations in Flexor Tendons,” The Jour- 
nal of Hand Surgery, Vol. 37, No. 2, 2011, pp. 217-223. http://dx.doi.org/10.1016/j.jhsa.2011.10.039

[14] J. R. Doyle, "Anatomy of the Flexor Tendon Sheath and Pulley System,” The Journal of Hand Surgery, Vol. 13, No. 4, 1988, pp. 473-484. http://dx.doi.org/10.1016/S0363-5023(88)80082-0

[15] H. Kato, A. Minami, N. Suenaga, N. Iwasaki and T. Kimura, "Long-Term Results after Primary Repairs of Zone 2 Flexor Tendon Lacerations in Children Younger than Age 6 Years,” Journal of Pediatric Orthopaedics, Vol. 22, No. 6, 2002, pp. 732-735. http://dx.doi.org/10.1097/01241398-200211000-00007

[16] J. M. Brunner, “The Zig-Zag Volar Digital Incision for Flexor Tendon Surgery," Plastic \& Reconstructive Surgery, Vol. 40, 1967, pp. 571-574. http://dx.doi.org/10.1097/00006534-196740060-00010

[17] R. S. Gill, B. H. Lim, R. A. Shatford, E. Toth, M. J. Voor and T. M. Tsai, "A Comparative Analysis of the Six Strand Double-Loop Flexor Tendon Repair and Three Other Techniques: A Human Cadaveric Study,” The Journal of Hand Surgery, Vol. 24, No. 6, 1999, pp. 1315-1322. http://dx.doi.org/10.1053/jhsu.1999.1315
[18] J. Nishida and P. C. Amadio, "Flexor Tendon Interaction after Pulley Reconstruction: A Biomechanical Study in a Human Model in Vivo," The Journal of Hand Surgery, Vol. 23, No. 4, 1998, pp. 665-672. http://dx.doi.org/10.1016/S0363-5023(98)80053-1

[19] S. W. Wolfe, R. N. Hotchkiss, W. C. Pederson and S. H. Kozin, "Green's Operative Hand Surgery," 6th Edition, Churchill Livingstone, New York, 2010.

[20] W. L. Newmeyer and P. R. Manske, "No Man's Land Revisited: The Primary Flexor Repair Controversy," The Journal of Hand Surgery, Vol. 29, No. 1, 2004, pp. 1-5. http://dx.doi.org/10.1016/S0363-5023(03)00381-2

[21] I. Kessler and F. Nissim, "Primary Repair without Immobilization of Flexor Tendon Division within the Digital Sheath: An Experimental and Clinical Study,” Acta Orthopaedica Scandinavica, Vol. 40, 1969, pp. 587-601. http://dx.doi.org/10.3109/17453676908989524

[22] S. J. O’Connell, M. M. Moore, J. W. Strickland, G. T. Frasier and P. C. Dell, "Results of Zone I and Zone II Flexor Tendon Repairs in Children,” The Journal of Hand Surgery, Vol. 19, No. 1, 1994, pp. 48-52. http://dx.doi.org/10.1016/0363-5023(94)90223-2 